\title{
Konstruktive Entwicklung einer industriell einsetzbaren Filter- HCA-Quelle für superharte Nanometer-Kohlenstoffschichten
}

\author{
Karin Jannasch, Peter Siemroth
}

\section{Einleitung}

Im Fraunhofer Institut für Werkstoff- und Strahltechnik Dresden wurde eine neuartige Plasmaquelle für die Abscheidung ultradünner und superharter Schutzschichten aus Kohlenstoff entwickelt, die ausgehend von einem aktuellen Anwendungsfall der Mikrosystemtechnik für die Zukunft ein breites potenzielles Einsatzfeld für Verschleißschutz- und reibmindernde Schichten besitzt. Für eine schnelle Umsetzung in die Industrie bieten sich vor allem kleine, innovative Unternehmen aus dem Umfeld als Partner an. Die Überfuihrung in professionelle Industrieanlagen erfordert allerdings eine umfassende ingenieurtechnische Überarbeitung, die häufig die Möglichkeiten junger Unternehmen übersteigt. So sind bei der Optimierung des Gesamtkonzeptes bzw. einzelner Baugruppen verschiedenste Faktoren wie die Eignung fuir den Dauerbetrieb, der Wartungsaufwand und die Produktionskosten zu berücksichtigen. An dieser kritischen Schnittstelle hat die Technische Fachhochschule Wildau (TFH Wildau) eine wichtige Rolle uibernommen und arbeitet in Form von Diplomthemen und Forschungspraktika für Hochschullehrer eng mit dem Fraunhofer Institut für Werkstoff- und Strahltechnik Dresden (IWS Dresden) zusammen.

\section{Der gepulste Hochstrombogen}

Die Bogenbeschichtung findet seit Jahren breiten Einsatz bei der Vergüitung von Werkzeugen und Bauteilen mit dünnen Hartstoffschichten, wie z. B. TiN, TiAlN, CrN, und hat die früher dominierende Sputtertechnik in vielen Bereichen verdrängt [1]. Der Vorteil des Vakuumbogens liegt in einem gegenüber den herkömmlichen Verfahren grundsätzlich anderen Mechanismus des Schichtwachstums. Die bogenerzeugten hochenergetischen Teilchen dringen in die obersten Atomlagen ein und bilden auf diese Weise eine eng mit der Unterlage verzahnte, dichte und glatte Schicht [2, 3]. Die erzielten Schichten zeichnen sich durch eine feinkristalline oder amorphe Struktur aus, Stängelwachstum tritt kaum auf. Damit lassen sich deutlich bessere Schutzwirkungen sowohl hinsichtlich Korrosion als auch bezuiglich Verschleiß erzielen. Ein relativ neues Schichtmaterial stellt der amorphe Kohlenstoff dar. Dessen strukturelle und mechanische Eigenschaften lassen sich in weiten Grenzen - zwischen relativ weichen polymerartigen und superharten wasserstofffreien Schichten - einstellen [4]. Gemeinsam ist allen amorphen Kohlenstoffschichten ein sehr niedriger Reibwert, der sie als tribologische Schutzschicht fuir viele Anwendungen eignet. Vakuumbogenabgeschiedene
Schichten zeichnen sich dabei durch einen besonders hohen Anteil von dreidimensionalen diamantartigen Kohlenstoffbindungen (sog. $\mathrm{sp}^{3}$-Bindungen) innerhalb der amorphen Matrix aus. Entsprechend einem $\mathrm{sp}^{3}$-Anteil von bis zu $70 \%$ kann auch die Härte $70 \%$ der des Diamanten erreichen [5]. Bei allen offensichtlichen Vorteilen hat die Vakuumbogenbeschichtung aber auch einen gravierenden Nachteil, der darin besteht, dass zwangsläufig gemeinsam mit dem hochaktivierten Plasma auch feste bzw. flüssige Partikel (meist als „droplets“ bezeichnet) emittiert und in die Schicht eingebaut werden [6]. Dies hat zur Folge, dass der Bogentechnologie derzeit noch potenzielle Anwendungsfelder verschlossen sind, wie die Beschichtung von Präzisionswerkzeugen, Schutzschichten in der Mikrosystemtechnik, Barriere- und Funktionsschichten der Mikroelektronik.

Als Lösungsweg bietet sich an, das leitfähige Plasma und die fast neutralen und schweren Partikel mittels magnetischer Felder voneinander zu separieren [7]. Dazu laufen seit vielen Jahren in aller Welt Laboranlagen, mit denen gezeigt werden konnte, dass es möglich ist, weitgehende Partikelfreiheit auf dem zu beschichtenden Substrat zu erreichen $[8,9,10]$. Nachteilig ist bei diesem Verfahren aber, dass die Abscheiderate durch Plasmaverluste erheblich reduziert wird (meist auf deutlich unter $20 \%$ ), so dass dieses Verfahren, außer für sehr wenige Spezialaufgaben, noch keine Anwendung gefunden hat $[12,13,14]$.

Nachdem Versuche in verschiedenen Forschungseinrichtungen gezeigt hatten, dass mittels gefilterter Bogenbeschichtung extrem gute Schichtqualitäten zu erreichen sind $[15,16,19]$, wurde zur Überwindung der Ratebeschränkungen im IWS Dresden ein gepulster Hochstrombogen (bezeichnet als „HCA“ = pulsed HighCurrent Arc) entwickelt. Im Gegensatz zu den derzeit noch standardmäßig verwendeten Gleichstrombögen handelt es sich beim HCA um eine Quelle, deren gemittelter Bogenstrom mit bis zu 1000 A ein Vielfaches des Bogenstroms von Gleichstromquellen (um $100 \mathrm{~A}$ ) beträgt [17]. Ein weiterer Vorteil liegt darin, dass generell weniger Droplets emittiert werden.

Darauf aufbauend entwickelte das IWS Dresden den Prototyp einer gefilterten HCA-Quelle, bezeichnet als $\Phi$ HCA für Beschichtungen in der Festplattenproduktion. Die Festplatte als ein besonders weit fortgeschrittenes Objekt der Mikrosystemtechnik vereinigt Komponenten, die mit klassischen Fertigungsverfahren hergestellt werden, mit Mikrosystemen von höchster Präzision. Schon jetzt beträgt der Abstand zwischen dem Schreib- 
/Lesekopf und der mit einer Geschwindigkeit bis über $200 \mathrm{~km} / \mathrm{h}$ darunter rotierenden Platte nur noch $10 \mathrm{~nm}$. Für die in Zukunft nötige Erhöhung der Speicherdichte ist die weitere Verringerung des Abstandes zwischen der magnetischen Speicherschicht und dem Kopf notwendig. Dafür werden Schutzschichten mit extremen Härten und guten Gleiteigenschaften in Schichtdicken unter $3 \mathrm{~nm}$ benötigt, die eine gute Haftung auf dem Untergrund haben, frei von Partikeln sind und die empfindlichen ferromagnetischen Schichten zuverlässig vor Korrosion schützen können. Kohlenstoffschichten in diesem Schichtdickenbereich können mit bisher eingesetzten Sputterverfahren nicht erreicht werden, mit dem gefilterten Bogen sind solche Schichten jedoch nachweislich realisierbar [16].

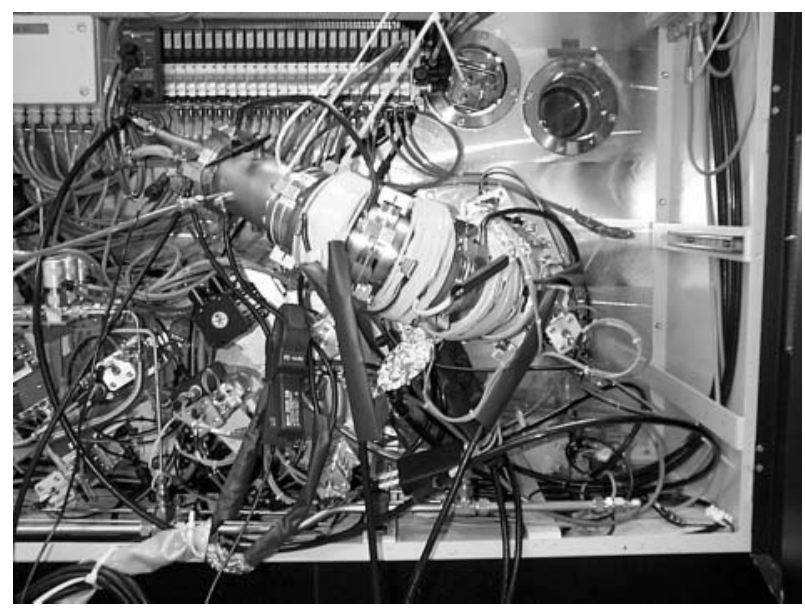

Abb. 1: Filter-HCA-Versuchsquelle zur Festplattenbeschichtung

Einsatztests mit dieser Quelle an einer industriellen Anlage zur Festplattenbeschichtung bei IBM in Mainz (Abb. 1) zeigten, dass mit der am IWS entwickelten gefilterten Pulsbogenquelle amorphe Kohlenstoffschichten abgeschieden werden können, die noch bei einer Dicke von 2 bis $3 \mathrm{~nm}$ ausreichend dicht, hart und korrosionsstabil sind, sowie extreme Glattheit, geringen Reibungskoeffizienten und gute chemische Beständigkeit aufweisen [17].

Wenn auch die geforderten Raten und Schichteigenschaften mit dieser Quelle realisiert werden konnten, so handelt es sich bisher erst um ein Versuchsmuster, dessen Überführung in eine industriell nutzbare Quelle zur Festplattenbeschichtung noch mit beträchtlichem Entwicklungsbedarf verbunden ist.
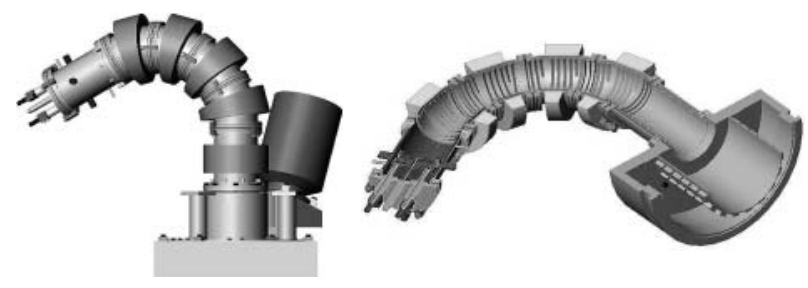

Abb. 2: Filter-HCA

Abbildung 2 zeigt den Aufbau der Quelle. Ein Target aus hochreinem Graphit befindet sich im oberen Teil der Quelle. Die Zündung der Bogenentladung erfolgt durch einen Hochspannungspuls. Die Zündelektrode befindet sich im Zentrum der Kathode. Die entstehenden Brennflecken bewegen sich nach der Zündung des Hochstrombogens von der Mitte der Kathode radial nach außen. Die Entladung wird abgebrochen, bevor die Brennflekken den Rand des Targets erreicht haben, und eine neue Entladung wird in der Mitte der Kathode gezündet. So bleibt der Materialabtrag auf die Targetfläche beschränkt. Das durch die Bogenentladung im Hochvakuum entstandene Plasma besteht aus nahezu vollständig ionisierten Kohlenstoff-Ionen mit einer Energieverteilung um $45 \mathrm{eV}$.

An den Bogenverdampfer schließt sich ein gekrümmter magnetischer Filter zur Separierung von Makropartikeln aus dem Plasmastrom an. Die Ionen des Plasmas werden im Magnetfeld der Spulen gefuihrt und im Winkel von $120^{\circ}$ abgelenkt, die weitestgehend ladungsneutralen Partikel werden dagegen vom Magnetfeld kaum beeinflusst und schlagen sich auf den Filterlamellen nieder. Dabei wird zwar die Abscheiderate verringert, aber die Partikelfreiheit auf dem zu beschichtenden Substrat garantiert.

Durch eine spezielle Struktur des Magnetfeldes direkt vor dem Substrat kann der stark gebündelte Plasmastrom aufgespalten und homogenisiert werden, so dass eine gleichmäßige Schichtdicke auf dem Substrat erreicht wird [11].

Die vorliegende Versuchsquelle kann an verschiedenste Reaktoren angeflanscht werden. Damit steht sowohl für umfassende Erprobungen in der Diskfertigung als auch für andere Anwendungen im High-Tech-Bereich, wie z. B. die Schreib-Lesekopfbeschichtung oder die Abscheidung von Kupferleitbahnen bzw. Barriere- oder Seed-Layer in der Schaltkreisfertigung, eine flexibel einsetzbare Versuchquelle zur Verfuigung. Mit ihrem modularen Aufbau kann diese Versuchsquelle auch schrittweise an neue Anwendungen angepasst, erprobt und optimiert werden.

\section{Aufgabenstellung}

Aus der industriellen Erprobung der vorhandenen Versuchsquelle und der daraus folgenden konzeptionellen Überarbeitung wurden die detaillierten Einzelaufgaben abgeleitet. Besondere Aufmerksamkeit muss den folgenden Problemen gewidmet werden:

- homogene Schichtabscheidung über die volle Fläche einer $95 \mathrm{~mm}$ Festplatte

- deutliche Erhöhung der Standzeit und Wartungsfreiheit

- schneller und unkomplizierter Targetwechsel

- Targetaufbereitung im laufenden System

- einfache Filterreinigung

- Reinraumtauglichkeit der Wartungsarbeiten

- thermische Stabilität aller Komponenten im Dauerbetrieb, Gestaltung und Auslegung der Kühlung

- Auswahl und Dimensionierung einzelner Komponenten

- fertigungsoptimierte Gestaltung des Filterbogens

- Anpassung an weitere Anwendungen

- Kostenabschätzungen 
Ein wesentlicher Fortschritt zur Lösung dieser Aufgaben wurden im Rahmen eines von Frau Jannasch am IWS Dresden und bei IBM Mainz durchgefuihrten Forschungspraktikums erzielt. Erste daraus resultierende Ergebnisse werden im Folgenden vorgestellt.

\section{Konstruktive Überarbeitung und Erprobung der Verdampfergeometrie}

\subsection{Ausgangssituation}
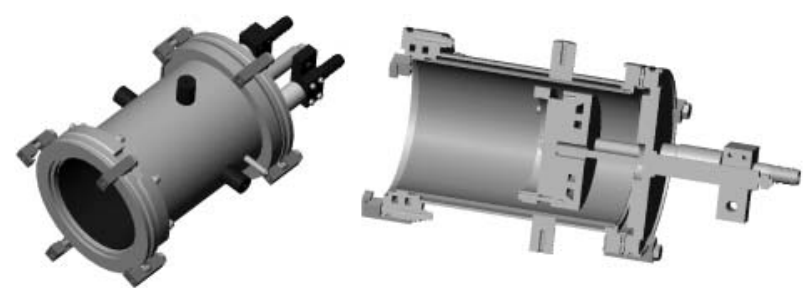

Abb. 3: Verdampfer der Versuchsquelle

Abbildung 3 zeigt den Verdampfer der Versuchsquelle. Das doppelwandige Gehäuse besteht aus einem Außenmantel aus Stahl und einem direkt gekühltem Innenrohr aus Kupfer, welches als Anode fungiert. Das runde Target ist mit einem Spannring auf einem gekühlten Targethalter aus Kupfer befestigt. Der Graphitring darüber deckt den Spannring aus Edelstahl ab und soll den Umfang des Targets gegen die Anode abschirmen. Der Zündstift ist im Zentrum des Targets angeordnet und wird mit einer Keramikdurchführung gehalten und gegen die Kathode isoliert.

Das Target hat eine Lebensdauer von 50000 Pulsen, das entspricht etwa 4 Stunden Festplattenbeschichtung, zeigt aber schon nach 10000 Pulsen bzw. 50 Minuten eine deutliche Kraterbildung. Infolgedessen verringert sich die Rate und es werden mehr Droplets emittiert. Besonders nachteilig ist die Beschichtung des Zündstiftes, die ebenfalls schon nach 10000 Entladungen zu Problemen bei der Zuindung der Pulse fuihrt.

Bei der jetzigen Laboranlage ist es für einen Targetwechsel oder auch nur für die Reinigung des Zündstiftes notwendig, die gesamte Anlage zu beliiften und den Verdampfer zu demontieren. Das bedingt jedes Mal eine längere Unterbrechung des Beschichtungsprozesses für die Beliuftung, Wartungsarbeiten und die anschließende Wiederherstellung des Vakuums.

Um die Mindestanforderungen für eine Einfuihrung in die Produktion erfüllen zu können, muss die Standzeit auf mindestens acht Stunden erhöht werden. Dazu wird ein automatischer Targetwechsel entwickelt, in dem auch eine Reinigung des Zündstiftes und eine eventuelle Aufarbeitung der Targetoberfläche durchgefuihrt werden kann. Hierzu liegt ein erster Entwurf der Fa. Weil über einen Targetwechsler vor, bei dem die Targets auf einer Trommel mit sechs Stationen angeordnet sind und innerhalb des Vakuums über einen Schrittmotor weiterbewegt werden.
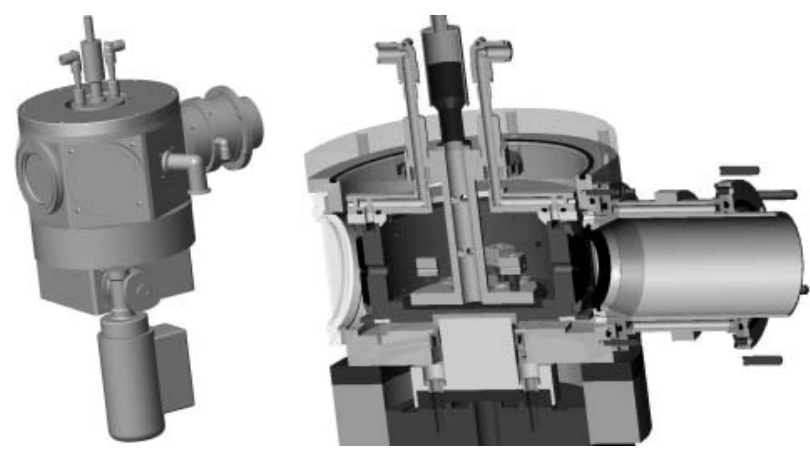

Abb. 4: Targetwechsler

Dieser Entwurf enthält einerseits eine ganze Reihe interessanter Lösungsansätze, die weiter verfolgt und überarbeitet werden müssen. Andererseits erscheinen einige Bereiche ungünstig für die Betriebssicherheit. Es muss geprüft werden, ob alle isolierenden Komponenten ausreichend vor einer Bedampfung geschützt sind, ob es zu parasitären Entladungen kommen kann, ob alle Baugruppen die notwendige mechanische Festigkeit aufweisen und welche Maßnahmen gegebenenfalls getroffen werden müssen, um diese Konstruktion zu verbessern. Dafür wurde die Verdampfergeometrie der vorliegenden Konstruktion fuir Versuche in einem Rezipienten modifiziert und durch neue Varianten ergänzt. Insbesondere ist die Anordnung des Targets, des Abdeckringes und der Anode kritisch und soll aus diesem Grunde variiert und getestet werden, während die eigentliche Dreh- und Wechseleinrichtung schon sehr gut gelöst ist.

Die damit verbundenen Veränderungen der Anordnung von Kathode und Anode dürfen sich nicht nachteilig auf die Ausbreitung der Brennflecken und die Verteilung des Plasmastromes auswirken. Dafür wurden fünf Gestaltungsvarianten entwickelt, als Versuchsmuster gebaut und in einer Vakuumkammer getestet.

\subsection{Varianten zur Verdampfergeometrie}

Abbildung 5 zeigt die prinzipielle Anordnung fuir Versuche in einem Rezipienten. Eine Kupfertrommel ist drehbar auf einem isolierenden Teflon-Stuitzring gelagert und hat am Umfang funf unterschiedlich gestaltete Stationen für die Aufnahme der Targets. Die massive Kupferanode kann in der Länge variabel aus zwei oder drei Elementen zusammengesetzt werden. Sie ist längs justierbar auf isolierenden Teflonfuißen gelagert und wird je nach Ausführungsvariante mit unterschiedlichen Abschlüssen versehen. Trommel und Anode sind massiv ausgefuihrt, damit innerhalb der einzelnen Versuchsreihen eine möglichst große Pulszahl ohne Überhitzung der Teile möglich ist.

Besonders die Übergänge zu isolierenden Bauteilen müssen so gestaltet sein, dass die Durchschlagsfestigkeit vergrößert wird und die wachsende Kohlenstoffschicht nicht zu schnell einen leitenden Überzug bilden kann. Aus diesem Grund sind an den kritischen Stellen Spalten mit Rillen zur Vergrößerung der Oberfläche vorgesehen. 

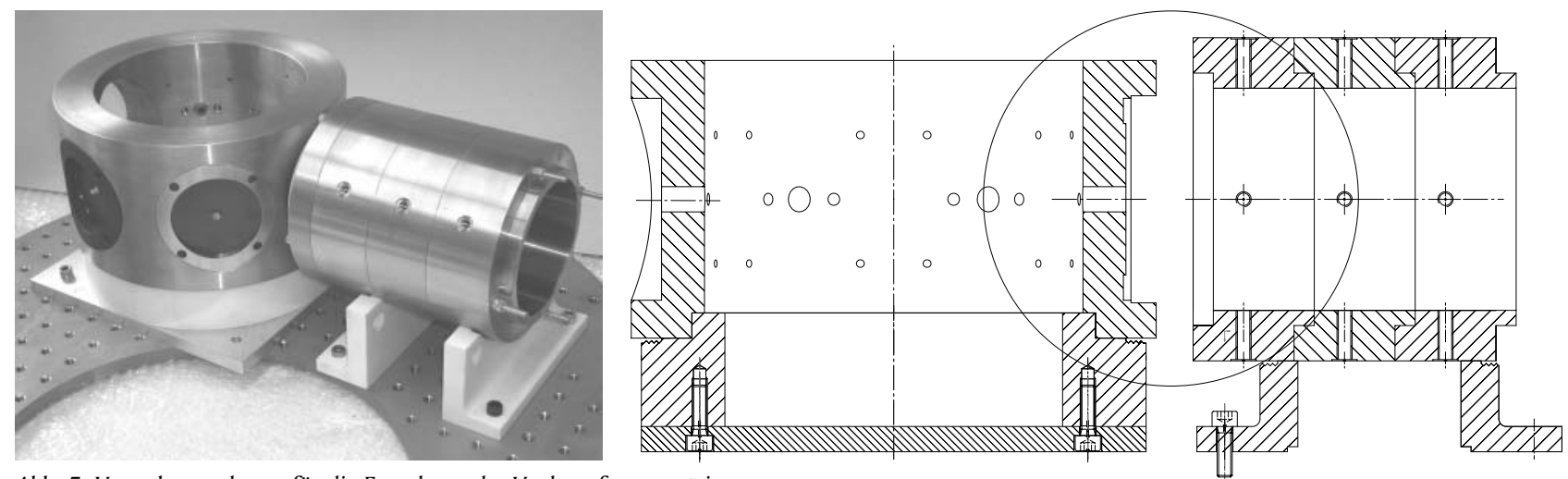

Abb. 5: Versuchsanordnung für die Erprobung der Verdampfergeometrie

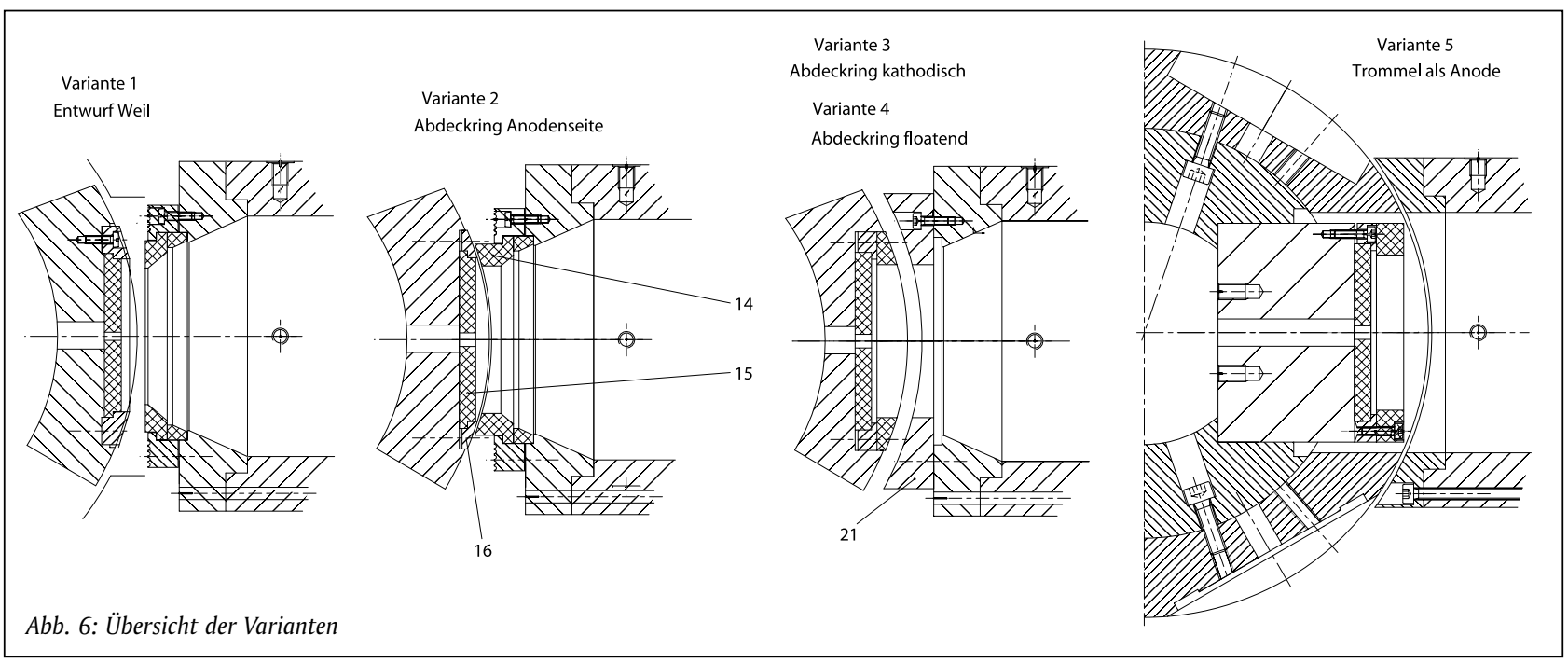

\section{Variante 1:}

Diese Anordnung entspricht weitgehend der vom Ingenieurbüro Weil entwickelten Konstruktion. Trommel, Target und Spannring bilden hier die Kathode. Der lichte Anodendurchmesser verjüngt sich. Der Abdeckring aus Graphit ist an der Öffnung der Anode befestigt und durch zwei Keramikringe gegen das Anodenpotenzial isoliert.

Problematisch erscheint, dass der Spalt zwischen Trommel (Kathode) und Abdeckring (floatend) aufgrund der Rundung der Trommel bis zu $7 \mathrm{~mm}$ groß wird. Dadurch kann seitlich Plasma austreten und unerwünschte Beschichtungen verursachen. Außerdem ist zu befuirchten, dass der große Abstand die Stabilität der Entladung verschlechtert. Die Brennflecken können bei entsprechender Lebensdauer nach außen bis auf den Targetspannring aus Metall laufen und dort Metallionen herauslösen, die zu unerwünschten Beimengungen in der Schicht führen. Mit den Versuchen soll geprüft werden, ob austretendes Plasma und von der Kathode herunterlaufende Kathodenflecken den Betrieb stören und ob die relativ feinen Komponenten aus Keramik ausreichend stabil sind.

\section{Variante 2:}

Der Targetspannring sowie der gegenüberliegende floatende Abdeckring auf der Anodenseite werden der Krümmung des Trommelaußendurchmessers angepasst und das Target weiter außen angeordnet. So können der Spalt zum Abdeckring und der seitliche Austritt von Plasma aus dem Spalt verringert werden. Mit dem geringeren $A b-$ stand des Targets zum floatenden Abdeckring und zur Anode wird der lichte Querschnitt größer und damit auch die wirksame Targetfläche. Aber auch hier besteht noch die Möglichkeit, dass der Bogen über das Target hinaus auf den Spannring und die Trommel zündet.

\section{Variante 3:}

Der ebene Targetspannring wird durch den unmittelbar aufgesetzten Abdeckring vor der Bogenerosion geschützt. Das entspricht der bisherigen Kathodenanordnung des Versuchsmoduls. Der Abdeckring liegt somit auch auf dem Kathodenpotenzial. Der Bogen hat damit aber auch die Möglichkeit, über den Abdeckring und darüber hinaus bis hin zur Kupfertrommel zu laufen. Wegen des geringen Abstandes zum Target ist bei dieser Variante der größte lichte Querschnitt am Abdeckring möglich. Abdeckring und Anodenende sind an den Trommelradius angepasst und erfordern nur einen kleinen Spalt, durch den nur geringe Plasmaverluste entweichen.

\section{Variante 4:}

Diese Variante unterscheidet sich von der vorherigen nur durch die Isolierung zwischen dem Spannring und dem floatenden Abdeckring. Damit sollten kaum noch Bogenspuren auf dem Abdeckring und der Trommel zu beobachten sein. Auch hier wird der Spalt durch abgerundete Formen auf Kathoden- und Anodenseite minimiert. Bei diesen beiden Varianten ist der Abstand vom Target zur Kupferanode deutlich geringer als bei den vorange- 
gangenen, weil hier der Platzbedarf für den Keramikring entfällt. Es besteht aber die Gefahr von Spannungsüberschlägen direkt von der Trommel (Kathode) auf die Anode.

\section{Variante 5:}

Bei dieser Variante wurde versucht, die bisherige, gut funktionierende Verdampferanordnung weitgehend beizubehalten. Ein Teil der Anode ist Bestandteil der drehbaren Trommel. Die zueinander drehbaren Elemente können bei angepasstem Trommelradius mit einem minimalen Spalt positioniert werden, da sie das gleiche Potenzial haben. Das Target ist bei dieser Versuchsanordnung auf einer Kupferhalterung angeordnet. Diese ist wegen der für eine Mindestanzahl Entladungen notwendigen Wärmekapazität sehr massiv ausgefuihrt. Für eine spätere Umsetzung sollte wieder eine gekuihlte Kathode zur Anwendung kommen, die weniger Platz benötigt und die Anordnung von mehreren Targets auf der Trommel erlaubt. Der Abdeckring kann in einfacher, ebener Form ausgefuihrt werden, da er vollständig innerhalb der Trommel versenkbar ist.

\subsection{Versuchsdurchführung}

Um die Versuchseinrichtung kostengünstig aufzubauen und die Versuchsdurchführung einfach zu gestalten, wurde auf eine Wasserkühlung der Komponenten verzichtet. Demzufolge begrenzt die durch die Entladungen eingebrachte Energie die Versuchsdauer. Abschätzungen hatten gezeigt, dass mehrere tausend Entladungen ohne Beliuftung möglich sein sollten. Aus den bisherigen Erfahrungen ist bekannt, dass insbesondere die Formierungsphase für das Betriebsverhalten einer Kathoden-AnodenGeometrie kritisch ist. Auf frischen Oberflächen (insbesondere der Kathode) breitet sich die Entladung viel schneller aus als auf solchen, die bereits längere Zeit bogenbehandelt sind. Damit besteht insbesondere in dieser Phase die Gefahr, dass außer dem Targetbereich auch andere Komponenten angegriffen werden. Nach etwa 500 Entladungen ist die Formierung abgeschlossen. Damit bietet die Versuchseinrichtung auch die Möglichkeit, die stationäre Arbeitsphase ausreichend zu untersuchen.

Für die Varianten 1, 2, 3 und 5 wurden Versuchsreihen mit vergleichbaren Einstellungen für verschiedene Sätze von Pulsblöcken entsprechend Tab. 1 durchgefuihrt, die Bogenentladungen auf dem Target fotografiert (Beispiele siehe Abb. 7) und der Verlauf von Strom und Spannung pro Puls mit dem Oszillographen (Beispiel siehe Abb. 8) aufgezeichnet. Gemessen wurden weiterhin für die Varianten 2, 3 und 5 die Temperaturen der einzelnen Bauteile und bei Variante 5 zusätzlich für einen beschichteten massiven Substratblock vor dem Versuch und der Verlauf der Abkühlung danach. Die Temperaturverläufe für eine der Varianten sind in Abbildung 16 zu sehen.

Im Anschluss wurden die Bauteile einer gründlichen Sichtkontrolle unterzogen und die Details fotografiert, wobei insbesondere auf Spuren von Fehlzuindungen sowie das Verlaufen des Bogens uiber das Target hinaus auf andere Bauteile geachtet wurde.

\begin{tabular}{|l|r|r|r|r|}
\hline Satz & $\begin{array}{r}\text { Strom } \\
\text { in A }\end{array}$ & $\begin{array}{r}\text { Pulsdauer } \\
\text { in } \mu \text { s }\end{array}$ & $\begin{array}{r}\text { Pulsfrequenz } \\
\text { in Hz }\end{array}$ & $\begin{array}{r}\text { Anzahl } \\
\text { Pulse }\end{array}$ \\
\hline 1. Formierung & 500 & 500 & 1 & 100 \\
\hline 2. Formierung & 1500 & 500 & 1 & 100 \\
\hline 3. Satz & 3000 & 500 & 1 & 100 \\
\hline 4. Satz & 3000 & 1000 & 1 & 100 \\
\hline 5. Satz & 3000 & 1400 & 1 & 100 \\
\hline 6. Satz & 3000 & 1400 & 50 & 1000 \\
\hline
\end{tabular}

Tabelle 1
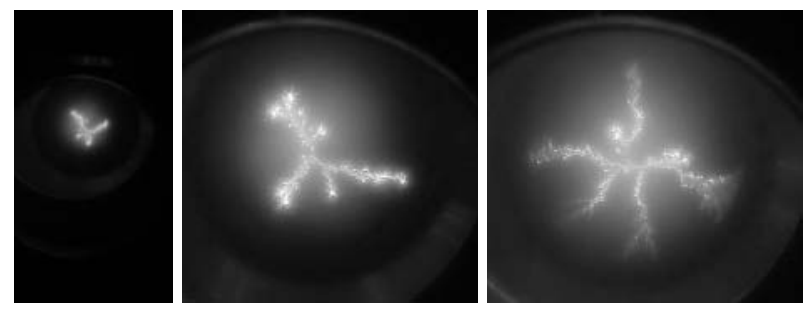

Abb. 7: Bogenentladungen aufKohlenstofftarget bei unterschiedlicher Pulsdauer
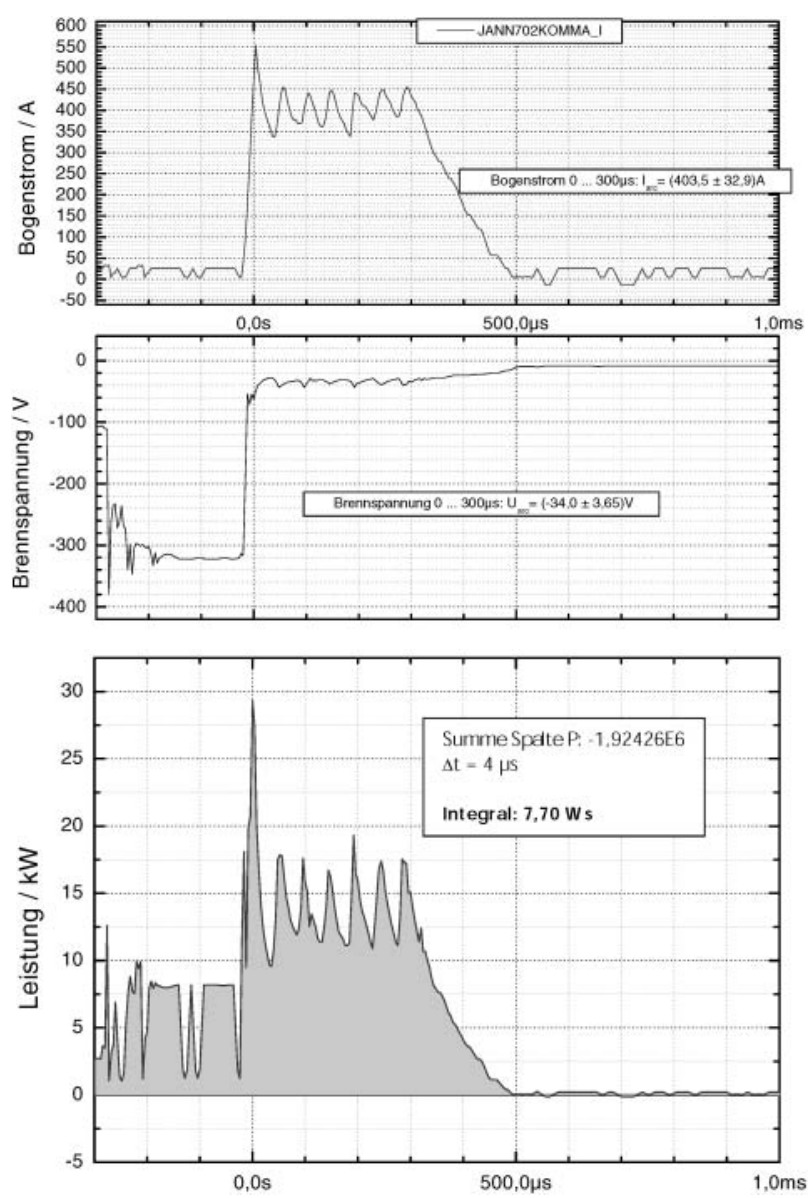

Abb. 8: Verlauf von Bogenstrom und Brennspannung über einen Puls

\subsection{Ergebnisse und Auswertung}

Bereits die wenigen durchgefuihrten Pulsblöcke zeigten, dass Variante 1 deutliche Mängel aufweist. So kam es schon nach wenigen Entladungen zum Bruch des Keramikringes. Deutlich erkennbar sind auch Überschläge auf die Verschraubung der Keramik, Bogenspuren auf dem Abdeckring und der Trommel. Im Oszillogramm sind extreme Spannungsspitzen aufgezeichnet. Die Bauteile, insbesondere der für die Isolierung wichtige Keramikring, zeigen deutliche Spuren der Beschichtung mit Stahl und Kupfer. 


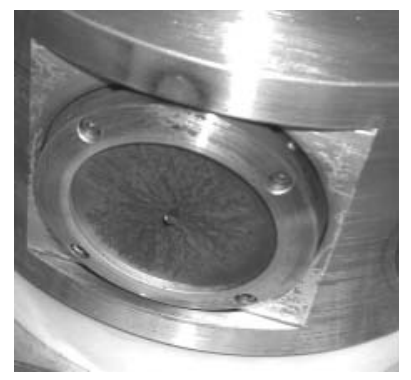

Abb. 9: Bogenspuren auf Trommel und Abdeckring

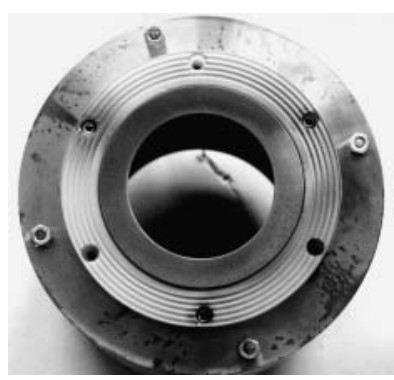

Abb. 10: Spuren auf Anode mit Keramikring
Variante 2 zeigt keine deutliche Verbesserung, auch hier sind tief eingegrabene Bogenspuren am Abdeckring, der Trommel und darüber hinaus sogar bis zum Abdeckring der Nachbarstation sowie Überschläge zur Anode im Bereich der Keramikbefestigung zu erkennen. Die geringere Breite des Spaltes selbst scheint wenig Einfluss zu haben, die Plasmaverluste an dieser Stelle sind unerheblich. Eine an dieser Stelle aufgestellte Glasplatte zeigt nach den Versuchen 1 und 2 fast keine Beschichtung. Die Wiederholung dieses Versuches mit größerem Spalt bestätigte diese Vermutung.

Bei Variante 3 sind noch Bogenspuren auf der Kupfertrommel erkennbar, aber nicht mehr so tief eingegraben wie bei den vorangegangenen Varianten (Abb. 11) Auf dem Spannring unter dem Abdeckring sind keine Bogenspuren erkennbar, ebenso keine Überschläge zur Anode. Die Stirnseite der Anode zeigt eine deutliche Kohlenstoffbeschichtung im Spaltbereich (Bild 12).

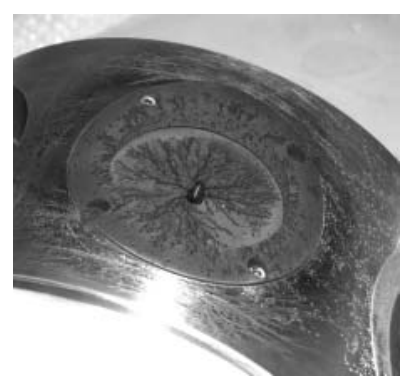

Abb. 11: Trommel

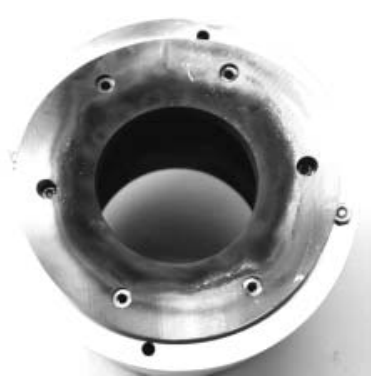

Abb. 12: Stirnseite Anode Variante 3
Die Variante 5 zeigt eine sehr gleichmäßige Brennspannung. Das ist ein deutlicher Hinweis darauf, dass hier kaum Bogenentladungen außerhalb des Targets aufgetreten sind. Tatsächlich machten alle Bauteile nach dem

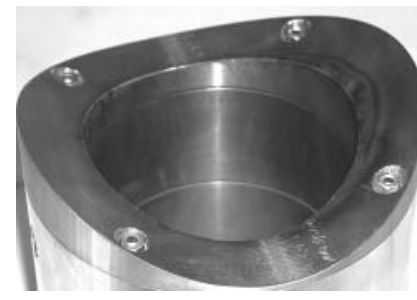

Abb. 13: Stirnseite Anode Variante 5 Versuch noch einen sehr guten Eindruck. Es sind keine Bogenspuren auf der Kupfertrommel oder dem Spannring erkennbar. Anodenseitig ist am Spalt kaum eine Kohlenstoffbeschichtung erkennbar (Abb. 13).

Bei keiner der untersuchten Varianten hat sich die Befürchtung bestätigt, dass Plasma in größeren Mengen seitlich durch den Spalt entweichen könnte. Die Verluste an dieser Stelle sind unerheblich. Die Beschichtung des Anodenrohres hat im ersten Drittel eine deutlich geringere Dicke (Abb. 14). Das ist neben den kaum erkennbaren Plasmaverlusten im Spalt ein Hinweis darauf, dass sich die Plasmawolke nicht allseitig, sondern vorzugsweise senkrecht vom Target weg ausbreitet und auch ohne fokussierende Magnetfelder schon in der Anode recht gut gebuindelt wird. Auch am Anodenausgang ist noch deutlich erkennbar, dass die Schichtdicke auf einer Glasplatte in der Mitte am höchsten ist (Abb. 15).

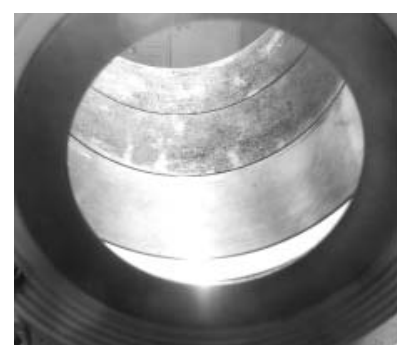

Abb. 14: Beschichtung innerhalb der Anode

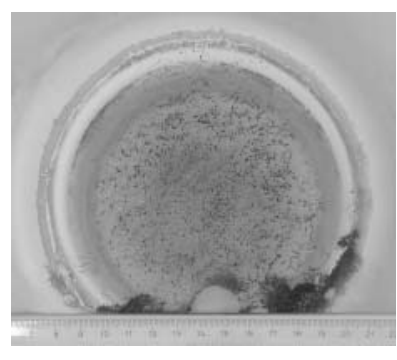

Abb. 15: Plasmaverteilung am Ausgang der Anode
Die Erwärmung der Bauteile (Abb. 16) ist geringer, als im Überschlag geschätzt wurde. Damit können weitere Versuchsreihen mit höheren Pulszahlen durchgeführt werden. Die in diesen Versuchsreihen erhaltenen Werte sind wesentlich genauer als früher gemessene und erlauben eine bessere Auslegung der Kuihlung bei der späteren Konstruktion eines industriellen Moduls.

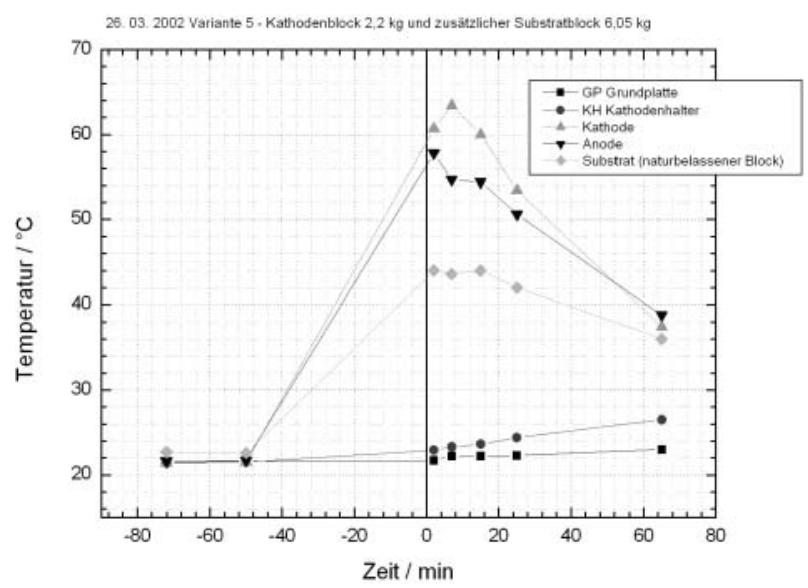

Abb. 16: Temperaturverlauf bei Variante $5 b$

Aus der Erwärmung der Bauteile (Abb. 17) im Vergleich mit der zugefuihrten elektrischen Leistung können erstmalig aussagekräftige Aussagen über die Spannungsanteile von Kathode, Anode und Plasma gemacht werden.

Während der Spannungsanteil über der Kathode annähernd konstant bleibt, zeigen sich insbesondere beim Anteil fuir das Plasma starke Schwankungen, die in weiteren Versuchen zu klären sind, auch eine Analyse der Schichteigenschaften kann dafuir Erklärungen geben.

Der Einsatz von Keramikbauteilen an thermisch hochbelasteten Stellen ist nicht vertretbar, hier kam es bereits nach wenigen Pulsblöcken zu Beschädigungen. Ungünstig stellen sich auch die Varianten dar, bei denen der Bogen die Möglichkeit hat, auf andere Materialien, wie z. B. Edelstahl oder Kupfer, zu laufen. Neben den Beschä- 


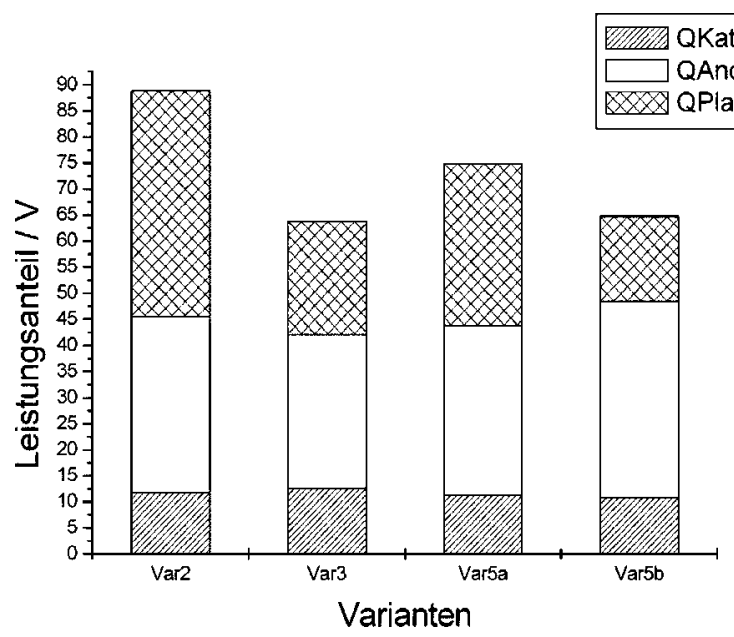

Abb. 17: Aufteilung der eingespeisten Energie zwischen Elektroden und Plasma

digungen und dem vorzeitigen Verschleiß der Bauteile selbst fuihrt das zur Verunreinigung des Plasmas, was sich für die späteren Schichteigenschaften sehr nachteilig auswirkt. Bei den Varianten 1 und 2 werden relativ häufig sehr hohe Spannungsspitzen induziert (teilweise weit über der Leerlaufspannung der Quelle), die zu Durchschlägen zwischen Kathode und Anode führen können und durch elektromagnetische Störstrahlung eine Gefährdung der umgebenden Elektronik darstellen.

Variante 5 zeigt insgesamt recht gute Ergebnisse, allerdings ist die Möglichkeit der Umsetzung von sechs Stationen auf der Trommel konstruktiv im Detail noch nicht untersucht, möglicherweise wird ein größerer Trommeldurchmesser oder die Begrenzung auf vier Stationen am Umfang erforderlich.

\section{Zusammenfassung}

Die ersten Versuche zeigen bereits deutlich die konstruktiven Schwächen des vorliegenden Entwurfes und geben Hinweise für die Überarbeitung des Targetwechslers. Um weitere Details bei der Bogenentladung zu verstehen und die Verdampfergeometrie noch besser optimieren zu können, müssen aber noch weitere Versuchsreihen insbesondere der Varianten 3 bis 5 mit deutlich mehr Pulszahlen und kleinen Modifikationen durchgefuihrt werden. Es ist vorgesehen, eine auf der Grundlage dieser Versuche vorgenommene Neugestaltung der Targetwechseleinrichtung als Patent anzumelden.

Insgesamt kann eingeschätzt werden, dass die Kombination der industriellen Erfahrung von IBM Mainz und der schicht- und plasmatechnologischen Kompetenzen des IWS Dresden mit dem Konstruktions-Know-how der TFH Wildau eine effektive Lösung der anspruchsvollen Aufgaben erwarten lässt und damit der Einfuihrung der Vakuumbogentechnologie in die Mikrosystemtechnik und Mikroelektronik den Weg ebnet.

\section{Danksagung}

Für die Möglichkeit der Durchfuihrung dieses Forschungspraktikums, die Bereitstellung von Versuchseinrichtungen und die umfassende fachliche Betreuung möchten wir dem IWS Dresden und der IBM Mainz herzlich danken. Die Arbeiten ordneten sich ein in das vom Bundesministerium für Bildung und Forschung geförderte Verbundprojekt „Innovative Reaktoren und in-situAnalytik für ultradünne Schutzschichten“ (FKZ-Nr. 13N7758). Besonderen Dank möchten die Autoren den Herren J. Berthold (IWS), B. Petereit, H. H. Schneider und H. Hilgers (alle IBM) fuir die aktive Unterstuitzung bei der gesamten Arbeit und viele nützliche Diskussionen aussprechen.

\section{Literatur}

[1] Rother, B. und Vetter, J.: Plasmabeschichtungsverfahren und Hartstoffschichten. Deutscher Verlag für Grundstoffindustrie, Leipzig 1992

[2] Besmond, J. H.: Fundamental Phenomena in Nucleation and Groth of Crystals. In: Chamberad, A., Hillrairet, J. (Eds.): Metallic Multilayers, Materials Science Forum 59\&60, (1990) $1-40$

[3] Schultrich, B.; Scheibe, H.-J.: Harte amorphe Kohlenstoffschichten. Jahrbuch Oberflächentechnik, Bd. 52, Hüthig Verlag, Heidelberg (1996) 109-129

[4] Frauenheim, T.; Jungnickel, G.; Köhler, T.; Stephan, U.: Structure and electronic properties of amorphous carbon: from semimetallic to insulating behaviour. J. Non-Crystalline Solids 182 (1995) 186-197

[5] Koskinen, J.; Schneider, D.; Ronkainen, H.; Muukkonen, T.; Varjus, S.; Burck, P.; Holmberg, K.; Scheibe, H.-J.: Microstructural changes in DLC films due to tribological contact. Surface and Coatings Technology 108-109 (1998) $385-390$

[6] Anders, A.; Anders, S.; Yu, K. M.; Yao, X. Y.; Brown, I. G.: On the macroparticle flux from vacuum arc cathode spots. IEEE Trans. Plasma Sci. 21 (1993), 440-446

[7] Aksenov, I. I.; Belous, V. A.; Padalka, V. G.; Khoroshikh, V. M.: Transport of Plasma Streams in a Curvilinear PlasmaOptics System. Sov. J. Plasma Phys. 4 (1978) 425-428

[8] Anders, S.; Anders, A.; Brown, I. G; MacGill, R. A.; Dickinson, M. R.: Vacuum arc ion source with filtered plasma for macroparticle-free implantation. Rev. Sci. Instrum. 65 (1994) 1319-1321

[9] Bendavid, A.; Martin, P. J.; Netterfield, R. P.; Kinder, T. J.: The properties of TiN films deposited by filtered arc evaporation. Surface and Coatings Technology, vol. 70, no. 1, (1994) 97-106

[10] Ben-Shalom, A.; Kaplan, L.; Boxman, R. L.; Goldsmith, S.; Nathan, M.: $\mathrm{SnO}_{2}$ transparent conductor films produced by filtered vacuum arc deposition. Thin Solid Films, vol. 236, no. 1-2, (1993) 20-26

[11] Anders, S.; MacGill, R. A.; Raoux, S.; Brown, I. G.: Modification of cathodic arc deposition profiles by magnetic multicusps. Proc. 17th Intern. Symp. Disch. El. Insul. In Vacuum - New York/USA, vol. 2, 1996, 909-913 
[12] Anders, A.; Anders, S.; Brown, I. G.: Focused injection of vacuum arc plasmas into curved magnetic filters. J. Appl. Phys., 75 (1994), 10, 4895-4899

[13] Gidalevich, E.; Boxman, R. L.; Goldsmith, S.: A simple method of enhancing magnetic filter efficiency. Proceedings ISDEIV. IEEE, vol. 2, (1998) 581-584

[14] Bendavid, A.; Martin, P. J.; Wang, X.; Wittling, M.; Kinder, T. J.: Deposition and modification of titanium nitride by ion assisted arc deposition. J. Vac. Sci. Technol. A, Vac. Surf. Films, 13 (1995) 1658-1664

[15] Anders, S.; Anders, A.; Bhatia, C. S.; Raoux, S.; Schneider, D.; Ager, J. W. III; Brown, I. G.: Properties of vacuum arc deposited amorphous hard carbon films. Proceedings of 3rd International Conference on the Applications of Diamond Films and Related Materials - 21-24 Aug. 1995. Gaithersburg, vol. 1, 1995, 809-812

[16] Singh-Bhatia, C.; Anders, S.; Brown, I. G.; Bobb, K.; Hsiao, R.; Bogy, D. B.: Ultra-thin overcoats for the head/disk interface tribology. Journal of Tribology, 120 (1998), 795799

[17] Siemroth, P.; Schülke, T.; Witke, T.; Schultrich, B.: Highcurrent arc - a new source for high rate deposition. Surface and Coatings technology 68-69 (1994) 314-319

[18] Neuhäuser, M.; Hilgers, H.; Witke, T.; Siemroth, P.: „Superharter Kohlenstoff abgeschieden mit gepulstem Hochstrombogen als Nanoschutzschicht für Magnetspeicherplatten“. Vakuum in Forschung und Praxis, 13 (2001) 215220

[19] Shi-Xu; Tay, B. K.; Tan, H. S.; Li-Zhong; Tu, Y. Q.; Silva, S. R. P.; Milne, W. I.: Properties of carbon ion deposited tetrahedral amorphous carbon films as a function of ion energy. Journal of Applied Physics, 79 (1996) 7234-7240

\section{Autoren}

\section{Prof. Dipl.-Ing. Karin Jannasch}

Technische Fachhochschule Wildau

FB Ingenieurwesen/Wirtschaftsingenieurwesen

Tel. (033 75) 508-231

E-Mail: jannasch@iwg.tfh-wildau.de

\section{Dr. rer. nat. Peter Siemroth}

Fraunhofer Institut für Werkstoff- und Strahltechnik Dresden

Abt. Dünnschichttechnologie

Winterbergstrasse 28

10277 Dresden

Tel. (03 51) 2583409

E-Mail: siemroth@iws.fhg.de 